\title{
Comparison of Two Neonatal Ischemic Injury Models Using Magnetic Resonance Imaging
}

\author{
STEPHEN ASHWAL, BEATRIZ TONE, HUI ROU TIAN, SAMUEL CHONG, AND ANDRE OBENAUS \\ Department of Pediatrics [S.A., B.T., H.R.T.], Department of Radiation Medicine [S.C., A.O.], Loma Linda University School of Medicine, \\ Loma Linda, CA 92320
}

\begin{abstract}
Using an 11.7-Tesla magnetic resonance imaging (MRI) scanner in 10-d-old rat pups we report on the evolution of injury over $28 \mathrm{~d}$ in a model of neonatal stroke (transient filament middle cerebral artery occlusion, tfMCAO) and a model of hypoxicischemic injury (Rice-Vannucci model, RVM). In both models, diffusion-weighted imaging (DWI) was more sensitive in the early detection of ischemia than T2-weighted imaging (T2WI). Injury volumes in both models were greater on $\mathrm{d} 1$ for DWI and $\mathrm{d} 3$ for T2WI, decreased over time and by d 28 T2WI injury volumes (tfMCAO $10.3 \%$ of ipsilateral hemisphere; RVM 23.9\%) were definable. The distribution of injury with tfMCAO was confined to the vascular territory of the middle cerebral artery and a definable core and penumbra evolved over time. Ischemic injury in the RVM was more generalized and greater in cortical regions. Contralateral hemispheric involvement was only observed in the RVM. Our findings demonstrate that high-field MRI over extended periods of time is possible in a small animal model of neonatal brain injury and that the tfMCAO model should be used for studies of neonatal stroke and that the RVM does not reflect the vascular distribution of injury seen with focal ischemia. (Pediatr Res 61: 9-14, 2007)
\end{abstract}

$\mathrm{C}$ urrent neuroprotective therapies of acute stroke in adults have not proven beneficial and because the delay in diagnosis of neonatal stroke is beyond the current window of opportunity for thrombolytic therapy, it is likely that future neonatal care will focus on delayed treatments implemented hours to days after ischemia. Temporally delayed treatment is even more likely in cases of neonatal ischemia as observable signs are more difficult to assess and observe. As such, being able to serially and noninvasively monitor the evolution of neonatal stroke in humans as well as animal models over weeks to months would be advantageous in studying mechanisms of injury, repair and responses to treatment. Magnetic resonance imaging (MRI) has greatly improved our ability to detect stroke in newborns but its use in neonatal stroke models has been limited by the technical challenges in serially acquiring such data $(1,2)$.

Received July 14, 2006; accepted August 17, 2006

Corresponding Author: Stephen Ashwal, M.D., Department of Pediatrics, Loma Linda University School of Medicine, 11175 Campus Street, Loma Linda, CA 92354, Tel. 909 558-8242, Fax. 909-558-0479; Email: sashwal@1lu.edu

Supported by the Pediatric Research Fund, Department of Pediatrics, Loma Linda University School of Medicine, grant number OSR\# 83018.

DOI: 10.1203/01.pdr.0000251612.16069.4b
The current study used high field strength (11.7T; Tesla) MRI to study the evolution of neonatal hypoxic-ischemic injury over a period of $28 \mathrm{~d}$ in two different models. We chose to compare a model of neonatal stroke (tfMCAO, transient filament middle cerebral artery occlusion) to that of the more commonly used model of neonatal hypoxic-ischemic brain injury (RVM, Rice-Vannucci model of unilateral carotid ligation and $1.5 \mathrm{~h}$ of hypoxia). We hypothesized that MRI could be used in a small animal model to serially and noninvasively monitor the evolution of injury over $28 \mathrm{~d}$ and that neuroimaging could elucidate temporal and spatial patterns of injury in the two models. Specifically, we wished to determine whether there were neuroimaging differences between the two models in: (1) the distribution of injury; (2) the degree of injury in striatum and cortex; (3) the evolution of core and penumbral injury; and (4) whether injury was confined to one hemisphere or involved the contralateral hemisphere.

\section{METHODS}

Ischemic injury induction. All treatment paradigms and experimental procedures were approved by the Loma Linda University Institutional Animal Care and Use Committee.

$\boldsymbol{t} \boldsymbol{A M C A O} \boldsymbol{O}$. Focal ischemia in the rat pup was accomplished using a filament model of temporary unilateral proximal occlusion of the left middle cerebral artery (MCA) as previously developed by our laboratory $(3,4)$. Unsexed 10 -d-old spontaneously hypertensive rat (SHR) pups $(14-18 \mathrm{gms} ; n=22)$ were anesthetized with $4 \%$ isoflurane and placed on a prewarmed pad (body temperature $=37^{\circ} \mathrm{C}$ ) on the platform of a Zeiss stereoscopic microscope (Model \# OMP1). Through a midline neck incision, three vessels were cauterized and a small incision made in the external carotid artery. A nylon filament (\#6.0, $0.07 \mathrm{~mm}$ ) was introduced through the incision and advanced into the internal carotid artery to block the MCA (20 min duration). Pups were allowed to recover in a normoxic environment and after $90 \mathrm{~min}$ of occlusion, they were checked for neurologic deficits before being reanesthetized.

$\boldsymbol{R} \boldsymbol{V} \boldsymbol{M}$. Five pups were studied using the standard RVM (5) of unilateral common carotid artery occlusion with $8 \%$ hypoxia. Unsexed 10-d-old SHR pups were prepared for surgery as above. However, with this model, the right common carotid artery of each pup was exposed, ligated (5-0 sutures) and the injured pups allowed to recover with their dams for $2 \mathrm{~h}$. They were then placed in a jar containing a humidified gas mixture (8\% oxygen-balance nitrogen at $37^{\circ} \mathrm{C}$ ) for $1.5 \mathrm{~h}$ and then allowed to recover.

MRI. MRI was performed in all animals at 1, 3, 7, 14, and $28 \mathrm{~d}$ after injury. These time points are commonly used endpoints in studies of ischemic brain

Abbreviations: DWI, diffusion-weighted imaging; FOV, field of view; MCA, middle cerebral artery; RVM, Rice-Vannucci model; tfMCAO, transient middle cerebral artery occlusion; SHR, spontaneously hypertensive rat 
injury in rat pup models $(1,2)$. We did not make more frequent measurements in the early period after ischemia as this study focused on demonstrating how neuroimaging may be used in a model of chronic injury and repair. The tfMCAO animals were imaged to verify successful occlusion of the MCA and this data were previously reported (4). The RVM pups were not imaged immediately after surgery because all pups had consistent volumes of injury in preliminary studies. No adverse respiratory, body temperature or heart rate changes were noted during the imaging.

Pups were lightly anesthetized using $1 \%$ isoflurane and body temperature was maintained at $37^{\circ} \mathrm{C}$ (4). MRI data were collected on a vertical Bruker Avance 11.7 T MRI ( $8.9 \mathrm{~cm}$ bore) with a $3.0 \mathrm{~cm}$ (ID) volume quadrature coil (Bruker Biospin, Billerica, MA). Two imaging data sets were acquired: 1) a 10 echo T2 (TR/TE $=4600 / 10.2 \mathrm{~ms}, 128^{2}$ matrix, $3 \mathrm{~cm}$ field of view (FOV), and 2 averages), and 2) a diffusion weighted study (TR/TE $=3000 / 25 \mathrm{~ms}$, two b-values $\left(0.72,1855.64 \mathrm{~s} / \mathrm{mm}^{2}\right), 128^{2}$ matrix, $3 \mathrm{~cm}$ FOV, and 2 averages). Each sequence collected 20 coronal slices $(1 \mathrm{~mm}$ thickness, interleaved 1 $\mathrm{mm})$. Due to age-related size increases, the 28-d imaging was done on a larger bore $(30 \mathrm{~cm})$ Bruker $4.7 \mathrm{~T}$ imager using the same parameters.

Volumetric image analysis. Region of interest (ROI) analysis on the T2 and diffusion weighted imaging (DWI) images was done using a 3D imaging program (Amira ${ }^{\mathrm{TM}}$, Mercury Computer Systems, Inc., Chelmsford, MA). Analysis was performed on the entire imaging T2 and DWI datasets to delineate spatial development of ischemic injury volume using visual thresholds. Analysis included extraction of injury volume (total; cortical and striatal; core and penumbra), nonischemic brain volume, and total brain volume, as previously described (4). Hemispheric ischemic injury was calculated over all slices (left hemisphere volume of lesion/left hemisphere total volume) and expressed as a percentage of the intact contralateral hemispheric volume.
Penumbral volumes were estimated at each time point by first measuring the core volume at $28 \mathrm{~d}$ using the $\mathrm{T} 2$ images. Based on the assumption that the total volume of injury could be partitioned into core and penumbral regions, the final core volume was subtracted from the total hemispheric volume of injury at $1,3,7,14$, and $28 \mathrm{~d}$ and then divided by the hemispheric volume to calculate the percent final volume (Table 1, Fig. 1).

Neuropathology. After the final MRI study on day 28, pups were deeply anesthetized (ketamine/xylazine: $90 / 10 \mathrm{mg} / \mathrm{kg}$ i.p.) and perfused transcardially with $10 \%$ buffered neutral formalin solution. Brains were removed, postfixed overnight and tissues were embedded in paraffin. $7 \mu \mathrm{m}$ thick sections cut from each block and mounted onto glass slides with every tenth section stained with cresyl violet according to our previously used protocol (4).

Statistical analysis. Significant differences in the temporal evolution of ischemic volume of each model were determined using a one-way Analysis of variance (ANOVA, SigmaStat) with a posthoc pair wise multiple comparison procedure (Tukey test), significant at $p<0.05$ and highly significant at $p<$ 0.01 for each time point. Regional volumes of ischemia (total, cortical and striatal) and sensitivity of T2 versus DWI were compared by $t$ tests $(p<0.05)$ using data from 3 and $28 \mathrm{~d}$.

\section{RESULTS}

Mean weight, percentage of animals surviving the injury, and the percent hemisphere injured with penumbral volumes from the DWI scores are summarized in Table 1. In the tfMCAO pups, survival at $3 \mathrm{~d}$ was $75 \%$ and decreased to $29 \%$

Table 1. Weight, \% survival, and \% hemispheric ischemic injury by model over time

\begin{tabular}{|c|c|c|c|c|c|}
\hline Model & 1 day & 3 days & 7 days & 14 days & 28 days \\
\hline \multicolumn{6}{|l|}{$t f \mathrm{MCAO}$} \\
\hline Number of Animals & 23 & 18 & 7 & 5 & 5 \\
\hline Avg. Weight (grams) & 15.4 & 15.4 & 18.8 & 42.4 & 96.6 \\
\hline$\%$ hemisphere ischemia by DWI & $46.3 \pm 5.4$ & $22.3 \pm 4.8$ & $7.8 \pm 2.1$ & $11.3 \pm 6.2$ & $10.4 \pm 5.6$ \\
\hline Estimated Penumbra Volume (\%) & 35.9 & 11.9 & -2.6 & 0.9 & 0.0 \\
\hline \multicolumn{6}{|l|}{ RVM } \\
\hline$\%$ survival & 100 & 100 & 80 & 80 & 80 \\
\hline$\%$ hemisphere ischemia by DWI & $43.6 \pm 4.6$ & $25.9 \pm 2.2$ & $15.0 \pm 1.1$ & $24.0 \pm 2.1$ & $15.6 \pm 4.5$ \\
\hline Estimated Penumbra Volume (\%) & 28.0 & 10.3 & -0.6 & 8.3 & 0.0 \\
\hline
\end{tabular}

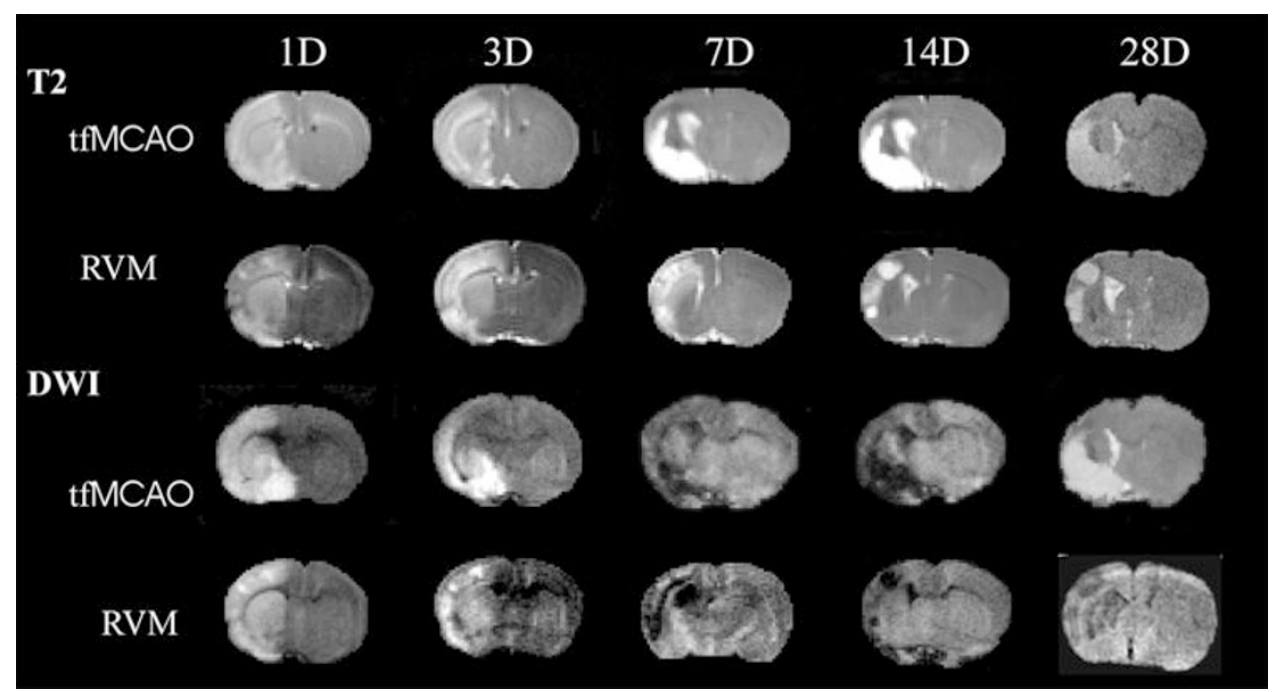

Figure 1. Comparison of T2WI and DWI in two models of ischemic injury. Injury is visualized at $1 \mathrm{~d}$ in both models of injury. However, T2WI signal intensity clearly peaks in the tfMCAO model at $3 \mathrm{~d}$ and is permanent by $7 \mathrm{~d}$. In the RVM animal the injury area is maximal at $1 \mathrm{~d}$ but these regions continue to evolve over the 14-28 d. At $28 \mathrm{~d}$ the tfMCAO model had cortical and striatal involvement whereas the RVM model had primarily cortical regions of injury. DWI changes were prominent at 1-3 d in both models. Within $1 \mathrm{~d}$ after tfMCAO, regions of high signal intensity (decreased diffusion), appeared in the infarcted regions that by $7 \mathrm{~d}$ converted to regions of dark signal intensity (increased diffusion). By $28 \mathrm{~d}$ these regions became bright again, indicative of T2 "shine through". DWI changes in the RVM model at $1 \mathrm{~d}$ involved the affected hemisphere, but by $3 \mathrm{~d}$ the cortex was the primary source of altered signal. Decreased signal intensity was observed primarily in the cortex at 7-28 d. 
by $7 \mathrm{~d}$. Many of the pups died because they were unable to eat and became dehydrated, despite routinely administered poststroke s.c. hydration (4). There was no evidence of infection. Twenty-one of the 29 tfMCAO pups that survived beyond $7 \mathrm{~d}$ lived until $28 \mathrm{~d}$. In contrast, survival in the RVM pups was $100 \%$ at $3 \mathrm{~d}$ and $80 \%$ at 7 to $28 \mathrm{~d}$. Despite the higher mortality rate, pups surviving tfMCAO had greater weight gains than the RVM pups but had similar ischemic injury volumes. No significant difference in infarct volume was found between animals that died or that survived to $28 \mathrm{~d}$.

MRI. tfMCAO ischemic changes were most apparent on DWI at $1 \mathrm{~d}$ after injury and subsided by $3 \mathrm{~d}$ whereas T2WI initially showed a large region of increased image intensity at these time points (Fig. 1). In contrast, the RVM model showed both diffuse $\mathrm{T} 2$ and DWI intensity changes at $1 \mathrm{~d}$ ay (Fig. 1). The area of T2 and DWI hyperintensities appeared maximal at $3 \mathrm{~d}$, with T2 hyperintensity continuing to evolve and more clearly demarcating the final lesion volume in both models where a distinct pattern of postnecrotic cortical and subcortical remodeling was evident. Changes in the DWI at 7 and $14 \mathrm{~d}$ were difficult to interpret because of ongoing cellular modifications and the development of necrotic injury. The hyperintensity seen at day 28 on DWI reflects a T2 'shine-through' effect rather than persistent cytotoxic edema. Evidence of the final ischemic injury observed on the T2 images lagged behind those seen with DWI.

Volumetric analysis. Volumetric analysis of the DWI showed a temporal alteration that was similar in both models (Fig. 2). On day 1, DWI volumes overestimated the final 28-d ischemic volumes. Over the first $7 \mathrm{~d}$, injury volumes decreased in both models with both models showing decreases in the percent hemisphere injured on day 1 compared with days 3-28 $(p=0.05)$. Notably, the 7-d injury volume was smaller in the tfMCAO than in the RVM $(p=0.02)$. DWI lesion volumes at $7 \mathrm{~d}$ were not significantly different from those at $28 \mathrm{~d}$ in either model, with final DWI volumes of injury being $10.3 \%$ (tfMCAO) and $15.6 \%$ (RVM) of the ipsilateral hemisphere (Fig. 2). Analysis of MR images from both models

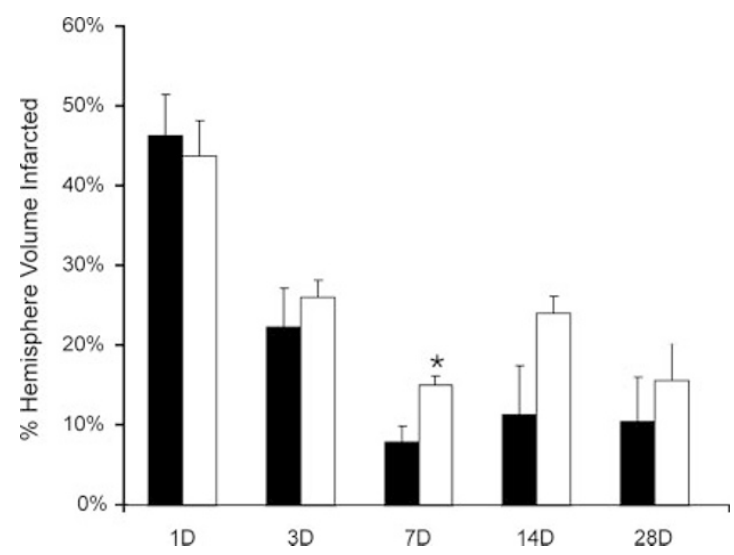

Figure 2. DWI comparison of infarct volume evolution in the tfMCAO and RVM models. DWI volumetric measurement of the \% hemisphere infracted in both models showed that the overall lesion volume decreased between 1 and $3 \mathrm{~d}$ and then stabilized from 7-28 d. At $7 \mathrm{~d}$, lesion volume is greater in the $\operatorname{RVM}\left(n=5\right.$, white bar) than $\operatorname{tfMCAO}(n=5$, black bar $)$ model $\left({ }^{*} p=0.02\right)$; this trend is also present at 14 and $28 \mathrm{~d}$.
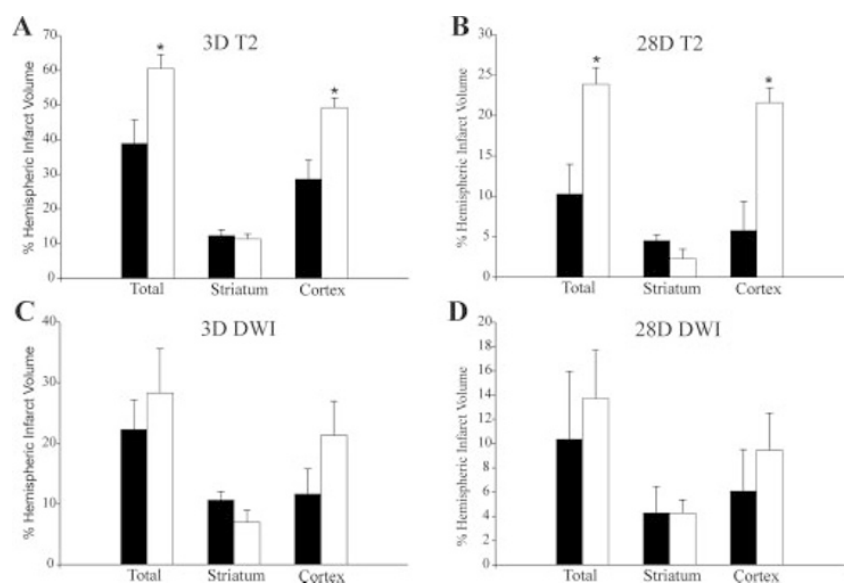

Figure 3. Comparison of $\mathrm{T} 2$ and DWI derived total hemispheric, striatal and cortical infarct volumes. Volumetric analysis demonstrates the cortical nature of the RVM model. (A) Comparison at $3 \mathrm{~d}$ using T2WI shows that the RVM $(n=5$, white bar) model encompasses a larger cortical stroke volume (20\% increase) compared with the tfMCAO ( $n=14$, black bars) pups. (B) At $28 \mathrm{~d}$ the relative cortical increases are further accentuated (RVM $n=5$, tfMCAO $n=5$ ). There is also a larger striatal infarct in tfMCAO pups. (C) DWI confirms the changes described in the $\mathrm{T} 2$ data (in $A$ ), but the striatal volumes are the same. $(D)$ At $28 \mathrm{~d}$ with DWI there are no significant differences in infarct volume in either model. $(* p<0.05)$

demonstrated regional changes that warranted a more thorough analysis (T2 and DWI), comparing cortical and subcortical structures.

Comparison of total hemispheric, cortical and striatal volumes from DWI and T2WI at 3 and $28 \mathrm{~d}$ are summarized in Fig. 3. After $3 \mathrm{~d}$ the percent hemispheric injury volume on the T2WI was significantly greater in the RVM $(60.6 \%)$ compared with the tfMCAO model $(38.9 \% ; p=0.08$; Fig. $3 A)$. The percent volume of cortical injury on T2WI was also greater in the RVM (49.2\%) than in the tfMCAO group $(26.6 \% ; p=$ $0.03)$. At $28 \mathrm{~d}$, the $\mathrm{T} 2$ derived cortical volumes remained greater in the RVM (21.6\% of hemisphere) than in the tfMCAO model $(5.8 \% ; p=0.009)$ (Fig. 3B). No significant difference between the models was seen in $\mathrm{T} 2$ derived striatal infarction volume at $28 \mathrm{~d}(p=0.13)$.

Figure 4 shows the three dimensional volume reconstructed T2 images (coronal, sagittal and angled) showing ischemic injury in the RVM and tfMCAO models at 3 and $28 \mathrm{~d}$. The RVM injury involves greater cortical areas (also see Fig. 1) that extend to the brain surface than was seen in the tfMCAO model. The ischemic injury in the RVM also involves brain regions that are more anterior and posterior than that induced by the tfMCAO, which more closely followed the limits of the vascular distribution of the MCA. When cortical involvement is present after tfMCAO, it includes more temporal regions compared with the parietal or retrosplenial cortical involvement in the RVM, possibly reflecting method of injury (occlusion versus hypoxia). The RVM also features: 1) greater overall cortical and subcortical injury volumes (Fig. 3, 5A), 2) contralateral injury due to global hypoxia (Fig. 5B, C), and iii) cortical injury that appears to be more laminar (Fig. $5 C$ ).

Ischemic core and penumbra differentiation. As shown in Fig. 1, the core and penumbral areas were more easily delineated in the tfMCAO model than in the RVM. Examination of 


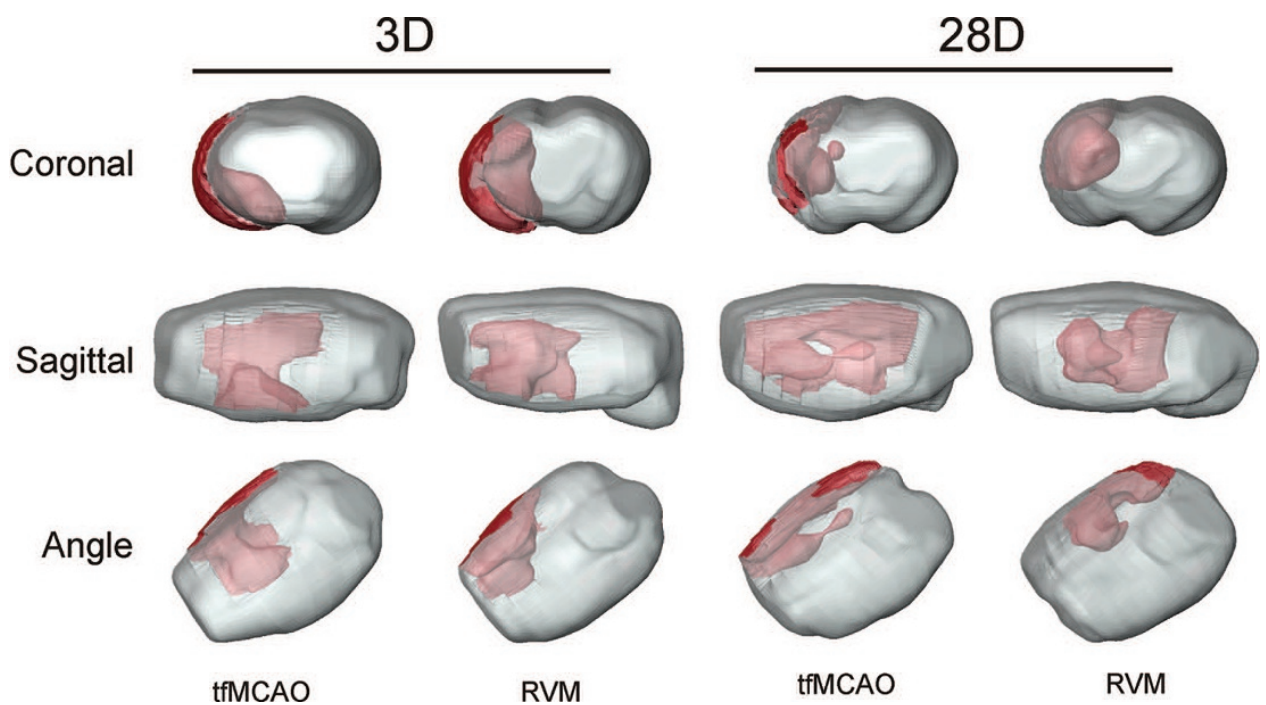

Figure 4. Volumetric comparisons of ischemic injury at 3 and $28 \mathrm{~d}$ in the RVM and tfMCAO. 3-Dimensional volumetric reconstructions from the same animals using T2WI at 3 and $28 \mathrm{~d}$ after infarct induction. The coronal, sagittal and angled images clearly show that the RVM animal has a larger rostral-caudal extent of injury than the tfMCAO animal at $3 \mathrm{~d}$. Furthermore, at $3 \mathrm{~d}$ the RVM has considerably more cortical involvement than the tfMCAO. At the final imaging point of $28 \mathrm{~d}$ both models showed similar volumes of damage, with the tfMCAO model being more anterior than the RVM. Again, the RVM had more cortical involvement than the tfMCAO animals (Total brain tissue Gray; infarcted tissue Red). the DWI at $1 \mathrm{~d}$ after tfMCAO shows an area of decreased diffusion (i.e., increased image brightness), similar in size to the area of injury observed in the $28 \mathrm{~d}$ T2 image, suggesting that this region may correspond to the area of irreversible tissue injury or ischemic core. There is also an adjacent area of altered signal intensity which resolves by $3 \mathrm{~d}$ and which does not overlap with any T2 abnormalities at $28 \mathrm{~d}$. There was similarly a large area of hyperintensity seen on the T2 images at $3 \mathrm{~d}$ that resolved by $28 \mathrm{~d}$. These areas of reversible diffusion restriction and $\mathrm{T} 2$ hyperintensity are likely to correspond to the ischemic penumbra.

Estimates of penumbral volumes are summarized in Table 1 and suggest that tfMCAO penumbra volumes were considerably larger than those in the RVM animals, even though the final infarct volume (core) was larger in the RVM.

Histologic validation of MRI findings. The histologic sections from the RVM pups appeared to encompass a larger portion of brain, involving striatum, hippocampus and cortex when compared with the tfMCAO pups (Fig. 6A, E). The tfMCAO pups had clear regions of necrosis in the retrosplenial cortical region (Fig. 6A, $C$ ) that were smaller and better defined than those found in the RVM pups (Fig. $6 F, G$ ). The contralateral hemisphere in tfMCAO pups did not show distinct pathology but rather normal-appearing neuronal and cellular histology (Fig. 6D, H).

\section{DISCUSSION}

Our previous report using an 11.7T scanner underscored the value of DWI and T2WI over $28 \mathrm{~d}$ to examine the evolution of neonatal stroke after tfMCAO and also correlated the imaging abnormalities with neuropathology (4). The present study demonstrates that there are clear neuroimaging differences in the volume and distribution of ischemic injury between the RVM and tfMCAO models and suggests that the RVM is better suited to the study of neonatal hypoxicischemic injury than it is to the study of neonatal stroke. As expected, the distribution of ischemic injury in the tfMCAO model was more clearly confined to the vascular territory of the MCA and a definable core and penumbra were present and evolved over time. In contrast, ischemic injury in the RVM was more generalized, had greater cortical involvement and involved the contralateral hemisphere.

We are unaware of any previous studies that compared neuropathological, imaging, and biochemical/metabolic derangements in adult or neonatal stroke models using tfMCAO versus RVM ischemic injury. Although it is likely that there are differences in the pathophysiology of ischemic injury, we did not explore this issue but confined our analysis to imaging and neuropathological data. The overall volumes of ischemic injury in the tfMCAO and RVM were similar but the distribution of injury was different (Figs. 2 and 3). These differences were best visualized with volumetric MR analysis (Figs. 2-4) than conventional neuropathology that is usually performed only on selected brain sections. The importance of determining differences in the distribution of injury in these two models is that different patterns of injury are likely to result in different long-term neurobehavioral, motor and structural outcomes as the pups mature. This is an important issue as animal-model research is more critically evaluating functional as well as neuropathological outcomes.

Long-term studies (i.e., 3 to $4 \mathrm{wk}$ ) have been performed in adult rat models showing the evolution of injury as measured by DWI and T2WI in the tfMCAO model (6) as well as in the Levine model (also unilateral carotid ligation with hypoxia) (7). Evidence of early injury was better seen with DWI although it overestimated final T2-derived injury volume. The DWI and T2WI changes that we observed are similar to those reported using lower field strength magnets in rat pup RVM (8) and stroke (9) models. Previous MRI studies have not gone beyond 3-7 d in either neonatal model; thus, the current study is the first to provide such comprehensive long-term data. DWI reflects development of acute cytotoxic edema due to cell membrane energy failure and T2WI primarily reflects vasogenic edema due to disruption of the blood brain barrier indicative of tissue at risk. The early appearance of $\mathrm{T} 2$ abnormalities in a specific region (e.g., white matter or hippocampus) would suggest that a particular brain region may have 


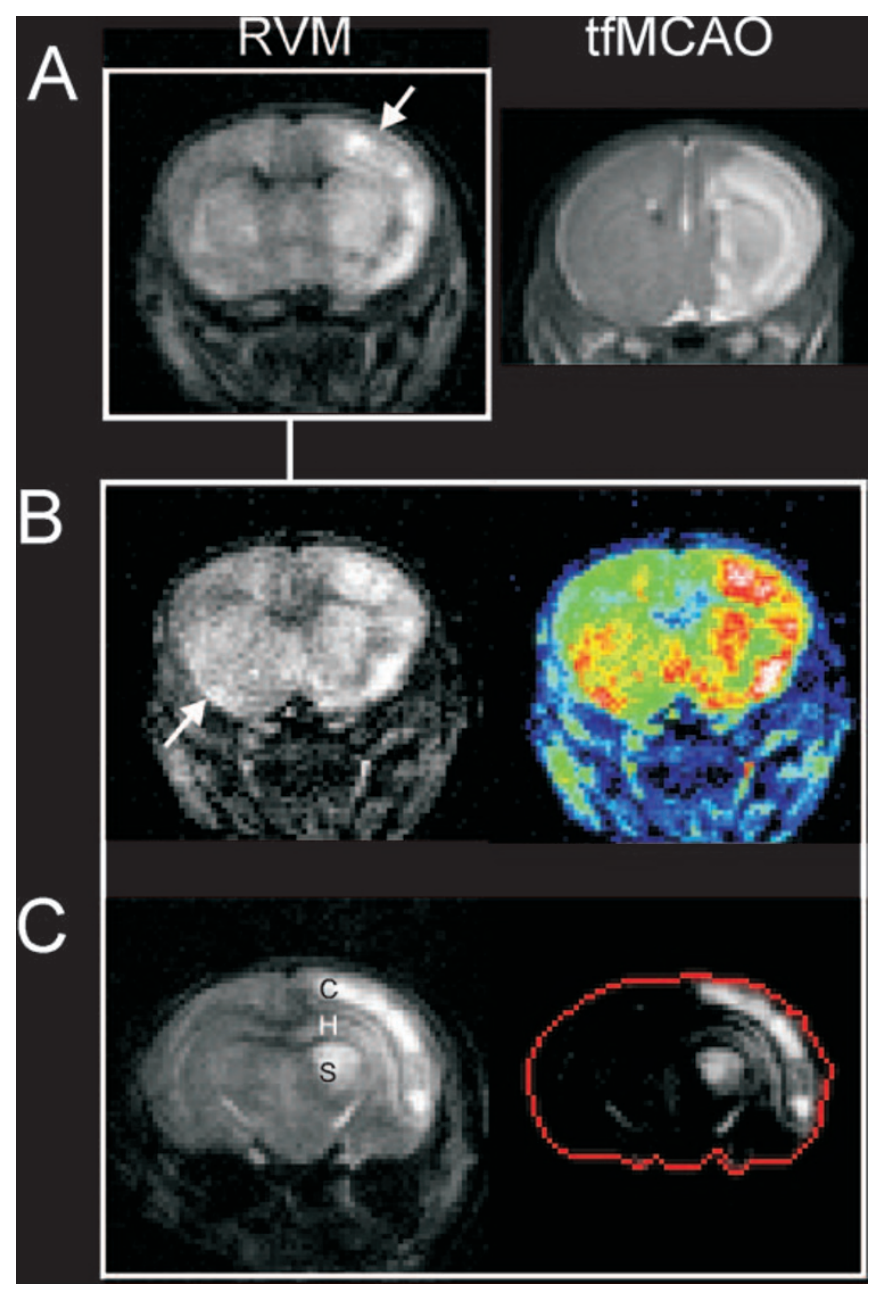

Figure 5. Cortical differences between the two models as determined by DWI. DWI at $3 \mathrm{~d}$ shows differential distribution of ischemic injury between tfMCAO and RVM. (A) DWI reveals the more extensive and defined cortical injury (arrow) in the RVM model $(n=5)$ compared with the tfMCAO $(n=$ 14), which shows more diffuse striatal injury at this time point. The distribution of injury in these models is more pronounced at latter time points (see Fig. 1). (B) An anterior RVM image shows that injury in this model may have some transient bilateral involvement (arrow). The pseudo-colored image highlights these altered DWI hyper-intensities on the contralateral side of this RVM pup. (C) A posterior slice in the RVM animal shows laminar cortical diffusion abnormalities that are clearly observed in the infarcted cortex (left image). Decreasing image thresholds further highlights the laminar diffusional changes within the cortex. ( $C=$ cortex, $H=$ hippocampus, $S=$ striatum).

suffered irreversible injury and would be less likely to respond to treatment (10).

Cortical, striatal and white matter injury in the two models. The RVM pups demonstrated greater superficial cortical injury as well as greater involvement of brain tissue outside the distribution of the MCA in the ipsilateral hemisphere (Fig. 4). The increased RVM lesion size likely is due to the more global effect of the $1.5 \mathrm{~h}$ of hypoxia resulting in increased vasogenic edema, to which T2 images are more sensitive. Striatal and white matter involvement was similar in both models. Based on the greater cortical involvement one might anticipate greater global cognitive impairments in the RVM. While, this is difficult to assess in rats, clinically it is well recognized that neonates with more diffuse hypoxic-ischemic injury are more likely to have cognitive impairments than those with stroke.
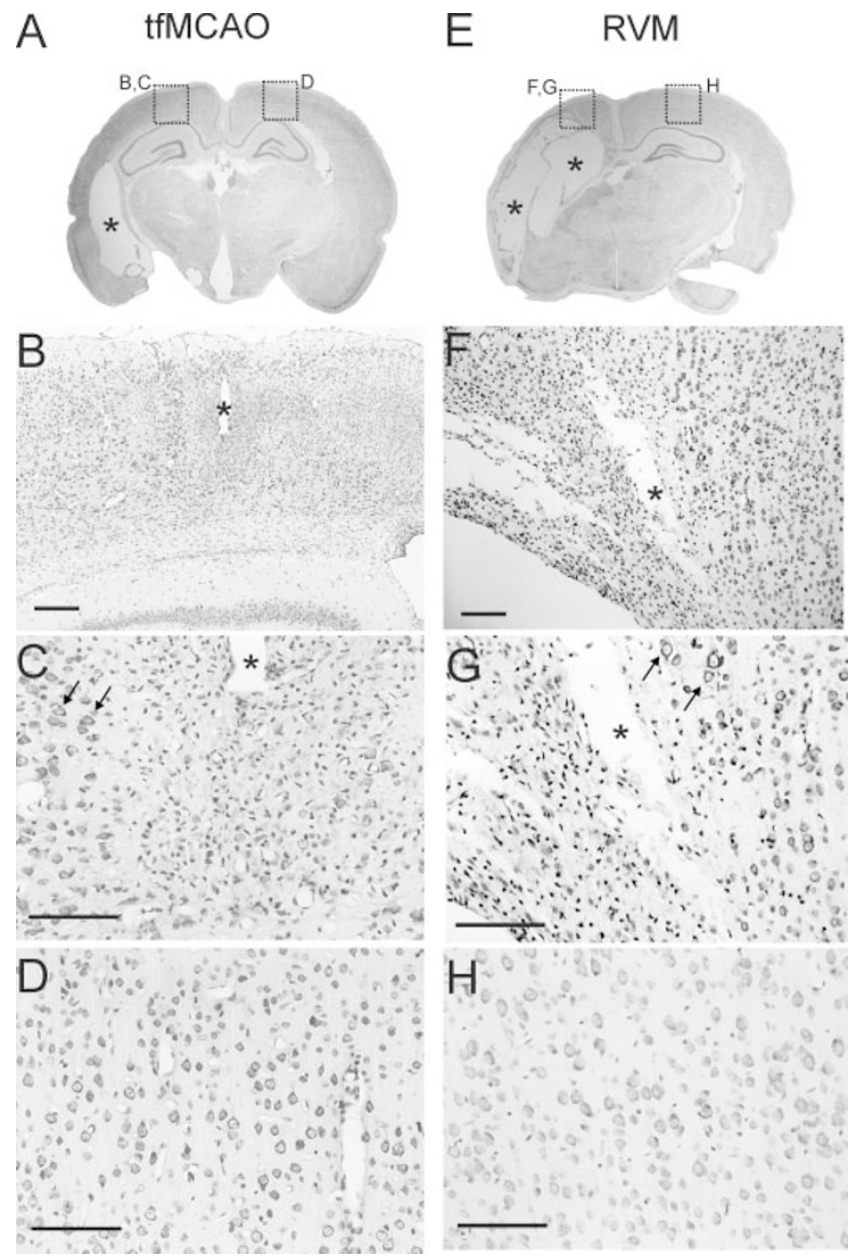

Figure 6. Histology of tfMCAO and RVM ischemic injury at $28 \mathrm{~d}$. Histologic sections from a $28 \mathrm{~d}$ animal after tfMCAO or RVM after cresyl violet staining. (A). An image from section at $28 \mathrm{~d}$ after tfMCAO. A low magnification $(1 \times)$ section clearly showing increased ventricle size. The labeled boxes indicate the regions selected for higher magnification. $(B)$ There are small regions of ischemic damage in the retrosplenial cortex. (*comparative regions) $(20 \times)$. (C) Many pyknotic neurons are seen in the injured cortex with normal appearing cells in the adjacent tissue (arrows). (40×). (D) In this section of the contralateral cortex there are numerous neuronal cell bodies with normal appearing density $(40 \times)$. (E) At $28 \mathrm{~d}$ after RVM there are larger regions of neuropathology. In this animal the striatum, hippocampus and much of the retrosplenial cortex is ablated as evidenced by increased ventricle size (*). (F) Numerous dead cells are observed at the ischemic border (*comparative regions) $(20 \times)$. (G) Many pyknotic cells are seen in the remaining cortex but in general the region is devoid of neuronal cell bodies $(40 \times)$. Occasional neuronal cell bodies are visible (arrows) but appear unhealthy compared with the contralateral cortex. $(H)$ In the contralateral cortex there are numerous neuronal cell bodies and normal appearing cell density $(40 \times)$. Calibration bar $=100 \mu \mathrm{m}$.

Previous reports (9) have shown that there is white matter involvement in neonatal stroke that is difficult to delineate in the neonate using MRI. However, the tfMCAO model appeared to have less corpus callosum involvement than the RVM model (Figs. 1 and 6). The sensitivity of white matter to hypoxic-ischemic injury is well known and thus the RVM model appears to have larger involvement that in large part may be due to the exposure to global hypoxia.

The mechanisms underlying differences in regional susceptibility are unclear but in part, are due to local variations in 
CBF during and after ischemia (7). In the RVM, changes in diffusion have been associated with secondary loss of highenergy phosphates (11) and the magnitude of this impairment correlates with injury severity (12). Since the duration of the cerebrovascular insult was similar in both models (i.e., $1.5 \mathrm{~h}$ ), the major differentiating features were the presence (RVM) or absence (tfMCAO) of an hypoxic insult, the obstruction of a larger (RVM) or smaller (tfMCAO) caliber artery, and the fact that in the RVM an inflow artery with a distal Circle of Willis is used whereas in the tfMCAO an end artery is occluded. Together, these differences would likely affect the ensuing metabolic cascade leading to cell death. Numerous other mechanisms have also been suggested for these regional susceptibility differences $(7,13)$.

As demonstrated in several recent MRI studies using the RVM, white matter injury appears earlier and after shorter periods of hypoxia than gray matter injury $(10,14)$, supporting earlier studies of increased susceptibility of this region (15). As shown in Figs. 1 and 6, the distribution of white matter injury is different in the RVM and tfMCAO models also suggesting different long-term motor and behavioral effects.

Core and penumbral regions in the two models. Evolution of injury in adult stroke models involving MCAO is described as containing a central primarily necrotic core and potentially salvageable penumbral regions. Imaging of the evolution of such injury is well-described in adult stroke models (16) and more recently in newborns $(9,17)$. Conversion of penumbral regions to irreversible injury is complex and multiple metabolic, inflammatory and apoptotic postreperfusion responses to injury have been described (18). In the current study, we demonstrated that DWI or T2WI in the tfMCAO model delineated a potentially salvageable penumbral region. Such imaging methods could serve as a biomarker of treatment effect. However, it would be necessary to compare mortality rates over time as it is possible that the evolution and volume of injury might be greater in pups that died.

Penumbral regions in the RVM likely exist although most researchers have refrained from characterizing the evolution of ischemic injury in such terms and there is virtually no published literature on this subject. Our study would suggest that the tfMCAO likely has a larger penumbra than the RVM model, even though the final infarct volume (core) is larger in the RVM. A recent study in the 7-d old rat pup defined 'penumbra-like' regions as an intermediate lesion based on tissue areas that showed enhanced GFAP-reactive astrocytes and spared cresyl-violet-stained neurons (19).

Contralateral hemispheric involvement. Neuroimaging showed increased restricted diffusion in the contralateral hemisphere in the RVM (Fig. 5). Although the RVM is often considered not to have contralateral involvement, the original description of the model in 1981 (5) and in several follow-up neuropathology studies $(20,21)$, described subtle contralateral cell injury, similar to that in our DWI/T2WI studies. In previous investigations of the RVM, the contralateral hemisphere has served as the control for measurement of various metabolic/gene regulatory mechanisms, an experimental design that our data suggest needs to be reconsidered.

Acknowledgments. The authors thank Dr. Yata Kenichiro and Dr. John Zhang for their assistance in the RVM experiments and Drs. David Michelson and Brenda Bartnik for their review of the manuscript.

\section{REFERENCES}

1. Ashwal S, Pearce WJ 2001 Animal models of neonatal stroke. Curr Opin Pediatr 13:506-516

2. Yager JY 2004 Animal models of hypoxic-ischemic brain damage in the newborn. Semin Pediatr Neurol 11:31-46

3. Ashwal S, Cole DJ, Osborne S, Osborne TN, Pearce WJ 1995 A new model of neonatal stroke: reversible middle cerebral artery occlusion in the rat pup. Pediatr Neurol 12:191-196

4. Ashwal S, Obenaus A, Tone B, Tian HR, Chong S 2006 Serial magnetic resonance imaging in a rat pup filament stroke model. Exp Neurol 202:294-301

5. Rice JE 3rd, Vannucci RC, Brierley JB 1981 The influence of immaturity on hypoxic-ischemic brain damage in the rat. Ann Neurol 9:131-141

6. Neumann-Haefelin T, Kastrup A, de Crespigny, A, Yenari MA, Ringer T, Sun GH, Moseley ME 2000 Serial MRI after transient focal cerebral ischemia in rats: dynamics of tissue injury, blood-brain barrier damage, and edema formation. Stroke 31:1965-1972

7. Dijkhuizen RM, Knollema S, van der Worp HB, Ter Horst GJ, De Wildt DJ, Berkelbach van der Sprenkel JW, Tulleken KA, Nicolay K 1998 Dynamics of cerebral tissue injury and perfusion after temporary hypoxia-ischemia in the rat: evidence for region-specific sensitivity and delayed damage. Stroke 29:695-704

8. Tuor UI, Kozlowski P, Del Bigio MR, Ramjiawan B, Su S, Malisza K, Saunders JK 1998 Diffusion- and T2-weighted increases in magnetic resonance images of immature brain during hypoxia-ischemia: transient reversal posthypoxia. Exp Neurol 150:321-328

9. Derugin N, Wendland M, Muramatsu K, Roberts TP, Gregory G, Ferriero DM, Vexler ZS 2000 Evolution of brain injury after transient middle cerebral artery occlusion in neonatal rats. Stroke 31:1752-1761

10. Meng S, Qiao M, Foniok T, Tuor UI 2005 White matter damage precedes that in gray matter despite similar magnetic resonance imaging changes following cerebral hypoxia-ischemia in neonatal rats. Exp Brain Res 166:56-60

11. Thornton JS, Ordidge RJ, Penrice J, Cady EB, Amess PN, Punwani S, Clemence M, Wyatt JS 1998 Temporal and anatomical variations of brain water apparent diffusion coefficient in perinatal cerebral hypoxic-ischemic injury: relationships to cerebral energy metabolism. Magn Reson Med 39:920-927

12. Blumberg RM, Cady EB, Wigglesworth JS, McKenzie JE, Edwards AD 1997 Relation between delayed impairment of cerebral energy metabolism and infarction following transient focal hypoxia-ischemia in the developing brain. Exp Brain Res 113:130-137

13. Vannucci RC, Vannucci SJ 2005 Perinatal hypoxic-ischemic brain damage: evolution of an animal model. Dev Neurosci 27:81-86

14. Qiao M, Meng S, Scobie K, Foniok T, Tuor UI 2004 Magnetic resonance imaging of differential gray versus white matter injury following a mild or moderate hypoxic-ischemic insult in neonatal rats. Neurosci Lett 368:332-336

15. McQuillen PS, Ferriero DM 2004 Selective vulnerability in the developing central nervous system. Pediatr Neurol 30:227-235

16. Bardutzky J, Shen Q, Bouley J, Sotak CH, Duong TQ, Fisher M 2005 Perfusion and diffusion imaging in acute focal cerebral ischemia: temporal vs. spatial resolution. Brain Res 1043:155-162

17. Manabat C, Han BH, Wendland M, Derugin N, Fox CK, Choi J, Holtzman DM, Ferriero DM, Vexler ZS 2003 Reperfusion differentially induces caspase-3 activation in ischemic core and penumbra after stroke in immature brain. Stroke 34:207213

18. Weinstein PR, Hong S, Sharp FR 2004 Molecular identification of the ischemic penumbra. Stroke 35:2666-2670

19. Felszeghy K, Banisadr G, Rostene W, Nyakas C, Haour FN 2004 Dexamethasone down regulates chemokine receptor CXCR4 and exerts neuroprotection against hypoxia/ischemia-induced brain injury in neonatal rats. Neuroimmunomodulation 11:404-413

20. Towfighi J, Zec N, Yager J, Housman C, Vannucci RC 1995 Temporal evolution of neuropathologic changes in an immature rat model of cerebral hypoxia: a light microscopic study. Acta Neuropathol (Berl) 90:375-386

21. Towfighi J, Mauger D, Vannucci RC, Vannucci SJ 1997 Influence of age on the cerebral lesions in an immature rat model of cerebral hypoxia-ischemia: a light microscopic study. Brain Res Dev Brain Res 100:149-160 